\title{
Spectral Gap Filling in Cognitive Networks: A Cooperative Game-Theoretic Approach
}

\author{
Chungang Yang, Student Member, IEEE, Jiandong Li, Senior Member, IEEE \\ (The State Key Lab. of ISN, Xidian University, Xi'an Shaanxi, 710071 China)
}

\begin{abstract}
An optimal joint channel selection and power control scheme is investigated in a cognitive network context, where the cognitive network is composed by multiple cognitive interference channels. Here, we take the fairness among multiple secondary users (SUs) and Pareto optimality measured by the capacity maximization into consideration. The complex cooperation and competition relationship among multiple SUs and primary users (PUs) is described with the refined signalto-interference plus noise (SINR) definition. According to the Nash axioms from the Nash bargaining cooperative game, the newly built utility function is formulated, and the spectral gapfilling problem is formulated as cognitive capacity Nash product maximization $(C C N P M)$. To improve the centralized algorithm design in in the cooperative game theory framework, we employ the dual decomposition technique to achieve the distributed bargaining approaches. The proposed approaches are with low implementation complexities and the little information exchange.

Index Terms-Cognitive network capacity; Cooperative game theory; Resource management; Power control; Decomposition technique; Network Utility Maximization.
\end{abstract}

\section{INTRODUCTION}

Cognitive networks hold tremendous promise for improving spectral utilization in mitigating the situation of shortage of spectrum. The celebrated cognitive radio technology as a promising paradigm is unified as an intelligent wireless communication concept that exploits side information about its cognitive context and their own capability, activity, channel information, code-books and other messages about other players [1]. Here, cognitive networks are wireless networks that consist of several types of users: often a primary user (PU) and secondary users (SUs). As [3] pointed out the study of the cognitive networks first coined in [4] is relatively new and many questions are yet to be answered, which composes of multiple coupled cognitive radio channels (CRCs).

Many attention has been focused on investigating resource management problems in the wireless networks, e.g., channel selection and power control in cognitive context [3]-[13]. To our best knowledge, most of the literature is dependent on the non-cooperative game and analyze the issue for the CR system or the CR-composed networks, e.g. [3] investigates the joint power control and channel allocation using the strategic non-cooperative game approach, [4] study the uplink pricing power control for a UWB-based cognitive radio system via Stackelberg game, the S-modular game is utilized in [5], [6], but [6] proposed a adaptive utility scheme, [7] is the potential game. We note that these are all based on noncooperative game, and other problems are mostly investigated using these similar model. But the celebrated Nash equilibrium solution (NES) for the non-cooperative game can't guarantee Pareto optimality, let alone the fairness among various SUs. Therefore, pricing based schemes are presented in [8], [9] to improve the overall performance and the Pareto optimality of NES. However, fairness criteria among SUs have never been guaranteed. To guarantee both the Pareto optimality and the fairness, the only way is to use cooperative game modeling framework, e.g. the Nash bargaining game [10], [12] for power control, [14] dynamic spectrum access, Kalai-Smorodinsky bargaining game [11] and the coalition game [13] for analyzing fair channel allocation in the OFDMA network. However, due to there are too much information to exchange, they either need a central controller [13]/central resource regulator [14], or the cooperation of multiple primary users (PUs) with SUs [16].

Due to the nice fairness and pareto efficiency properties, the cooperative game theoretical framework is still a promising technique. In this paper, we formulate spectral gap-filling problem using the Nash bargaining cooperative game model, but to design the distributive bargaining process with limit information exchange. What we are concern is also different from aforementioned literatures. We investigate cooperation/competition relationship among multiple SUs and PUs. Based on the celebrated iterative water-filling algorithm (IWFA), we propose two distributed bargaining approaches for search for the Nash bargaining solution (NBS).

\section{SYSTEM MODEL AND PROBLEM FORMULATION}

\section{A. System Model}

We investigate the cognitive network, which is composed by the $N$ secondary users and $M$ primary users. We assume that each primary user will share his own spectrum band $k \in K$ with the $N$ SUs. So there are $K$ channels provided by the $M$ PUs to share with the $N$ SUs in the cognitive network. Both the PUs and the SUs care about the terminal-to-terminal performance shown as Fig.1.

Here $g_{i, j}, i, j \in N$, and $h_{m, i}, m \in M, i \in N$ are the cognitive channel gain. When $i=j, g_{i, i}$ represents the communication channel gain, otherwise, it represents the interference channel gain. $h_{m, i}$ is the interference gain from the ist SU-transmitter (SU-Tx) to the mst PU-receiver (PU$\mathrm{Rx}$ ). In this paper, we focus on how the SU chooses his power level to maximize the cognitive network capacity but with the performance of the PU into consideration. Meanwhile, we pay attention to the cooperation and the competition among them, 


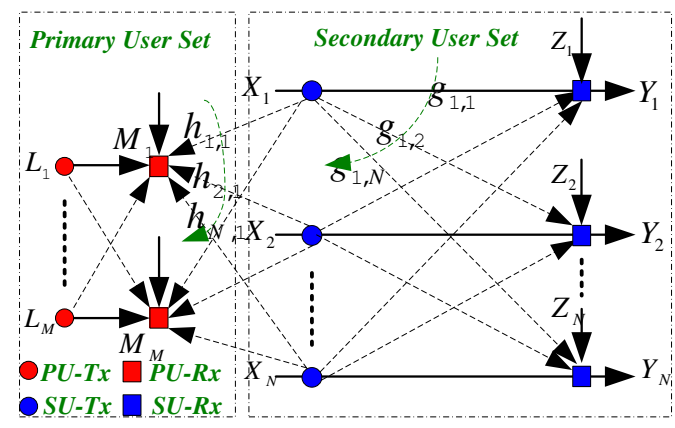

Fig. 1. System model.

which will reflect in the new form of signal-to-interference plus noise (SINR) definition.

\section{B. Redefined SINR}

To the knowledge of our best, most of the previous work did not seriously consider the SINR definition issue. They usually take the form of the traditional definition, without the consideration of the interference from the primary to the secondary user. In [8], where the authors consider this constraint, but fail to describe the complex interference relationship. It is necessary to redefine the SINR, motivated by the multitone sharing problem research in [17]. We denote SINR in the cognitive spectrum sharing scenario as (1).

Here $p_{i}(k)$ is the transmission power level of $S U_{i}$ on the channel $k,\left|g_{i, j}(k)\right|^{2}$ is the channel gain between the transmitter of $S U_{i}$ to the receiver of $S U_{j}$, and when $i=j$, it represents the communication link gain, when $i \neq j$ represents the interference link gain. $p_{m}\left(k_{1}\right)$ is the transmission power level of $P U_{m}$ on the channel $k_{1} \cdot\left|h_{m, i}\left(k_{1}\right)\right|^{2}$ is the corresponding channel gain from the transmitter of $P U_{m}$ to the receiver of the $S U_{i}$.

Here, we should be particularly emphasized mutual interference factor denoted as $\rho(\Delta k)$,

$\rho(\Delta k)=\left\{\begin{array}{l}1, \Delta k=0, \\ \frac{2}{K^{2} \sin ^{2}\left(\frac{\pi}{K} \Delta k\right)},-(K-1) \leq \Delta k \leq(K+1), \Delta k \neq 0 .\end{array}\right.$

It can well depict the interference relationship. There is such a fact that when different user access the same channel, they will appear in varying degrees of performance loss if there isn't one better, preassigned sharing strategies to achieve rationalely scheduled. The relationship can well reflected by the channel sharing policy. For example, when $\rho(\Delta k)=0$, there are two users conflict with each other where sharing the same channel, it is the total cooperation case and $\rho(\Delta k) \neq 0$, it represents the partial cooperation case.

\section{Utility Function Design}

Throughout this paper, all users want to maximize the overall performance of capacity. Also, from the cooperative game perspective, we intend to employ the NBS fairness as the fairness criteria.
Corollary 1: The unique, Pareto optimal and fair solution for the NBS game can obtained by maximizing the payoff function takes the form of the Nash Product, shown as

$$
V=\prod_{i} U_{i}^{\omega_{i}}\left(P_{i}, P_{-i}\right)
$$

where $\omega_{i}$ is the weight factor.

If the individual utility is $U_{i}^{k}\left(P_{i}, P_{-i}\right)=\gamma_{i}^{k}\left(P_{i}, P_{-i}\right)$, now the total performance measurement under the Corollary 1 is $V=\coprod_{i} \gamma_{i}^{\omega_{i}}\left(P_{i}, P_{-i}\right)$, where $\gamma_{i}$ is

$$
\gamma_{i}\left(p_{i}, p_{-i}\right)=\prod_{k} \frac{p_{i}(k)\left|g_{i, i}(k)\right|^{2}}{\xi_{i}(k)}
$$

if we use $\xi_{i}(k)$ is the original definition of SINR in (1), where $\xi_{i}(k)$ is replaced by (5).

Here $S U_{i}$ always expects to attain the optimal and fair sharing on the multiple channels. (5) satisfies the Corollary 1, too. Maximizing the Nash Product function has been proved to be the only way for pursuing the overall performance improvement and the fairness amongst multiple users [18].

To sum up, at this time the payoff function defined in (3) can be expressed as

$$
\begin{aligned}
& V=\prod_{i} \gamma_{i}^{\omega_{i}}\left(p_{i}, p_{-i}\right) \\
& =\prod_{i}\left(\prod_{k} \frac{p_{i}(k)\left|g_{i, i}(k)\right|^{2}}{\xi_{i}(k)}\right)^{\omega_{i}}
\end{aligned}
$$

Meanwhile, we give another corollary, although it have repeatedly appeared in the literature, e.g., [11]-[13], [20].

Corollary 2: The Nash bargaining solutions is always obtained using the log form payoff function, and we can prove it satisfies the proportional fairness [11]-[13], [20].

Especially, under the log-transformation in (2) the problem is equivalent transformation, so the solution does not undergo any change in the nature of fairness and Pareto efficiency. We give the equivalent transformation theorem and further in the framework of cooperative game theory, the payoff function in (6) that can be equivalently defined.

Theorem 1: The payoff function in (6) is equivalent to

$$
V=\sum_{i} \sum_{k} \omega_{i} \log _{2}\left(\frac{p_{i}(k)\left|g_{i, i}(k)\right|^{2}}{\xi_{i}(k)}\right)
$$

The certification process have been omitted here, which can be found in our previous work [11].

From the cooperative game-theoretic perspective, we have well considered the requirements of Game Theory. This form of payoff function design is to meet the Nash Axioms.

Here, we must also discuss and take into account the physical communication meaning of the payoff function. Inspired by the question itself in this paper,we have

$$
V=\sum_{i} \sum_{k} \omega_{i} \log _{2}\left(1+\Gamma \frac{p_{i}(k)\left|g_{i, i}(k)\right|^{2}}{\xi_{i}(k)}\right)
$$

Although, on the surface, such a transformation may only change on the value. It is only added with a constant "1", and then multiplied by a factor $\Gamma$. In fact, these can be reflected in value of the original definition of $\frac{p_{i}(k)\left|g_{i, i}(k)\right|^{2}}{\xi_{i}(k)}$. 


$$
\begin{gathered}
\gamma_{i}(k)=\frac{p_{i}(k)\left|g_{i, i}(k)\right|^{2}}{\sum_{j=1, j \neq i}^{N}\left(\sum_{k_{1}=1}^{K} \rho\left(k-k_{1}\right) p_{j}\left(k_{1}\right)\left|g_{j, i}\left(k_{1}\right)\right|^{2}\right)+\sum_{m=1}^{M}\left(\sum_{k_{1}=1}^{K} \rho\left(k-k_{1}\right) p_{m}\left(k_{1}\right)\left|h_{m, i}\left(k_{1}\right)\right|^{2}\right)+\sigma_{i}^{2}(k)} \\
\xi_{i}(k)=\sum_{j=1, j \neq i}^{N}\left(\sum_{k_{1}=1}^{K} \rho\left(k-k_{1}\right) p_{j}\left(k_{1}\right)\left|g_{j, i}\left(k_{1}\right)\right|^{2}\right)+\sum_{m=1}^{M}\left(\sum_{k_{1}=1}^{K} \rho\left(k-k_{1}\right) p_{m}\left(k_{1}\right)\left|h_{m, i}\left(k_{1}\right)\right|^{2}\right)+\sigma_{i}^{2}(k) .
\end{gathered}
$$

\section{Multiple Constraint Conditions}

For this spectrum sharing problem in the cognitive context, there are many limitations and constraints. In addition to the restrictions and requirements from cognitive radio itself, there are factors that need to be considered in game theory.

First of all, we are concerned about restrictions on game theory, as

$$
C_{i, \min } \leq \sum_{k=1}^{K} \log _{2}\left(1+\Gamma \frac{p_{i}(k)\left|g_{i, i}(k)\right|^{2}}{\xi_{i}(k)}\right), \forall i
$$

where $C_{i, \min }$ is the minimum capacity requirement. In other words, for each user, while ensuring the overall utility maximization, we must also take into account the usefulness of the individual utility. This restriction, in the current study, is not very common. In cooperative game theory, $C_{i, \min }$ is called the disagreement point, which is the Nash equilibrium solution and is the start-point of the Nash bargaining. That is to say, if multiple users can't achieve the practical agreements. They will use this strategy. This value also changes with the changes in the cognitive environment.

Second, from the cognitive network, as the interferencepower constraints, we deal with two catteries of constraints: the peak interference-power constraints from PUs:

$$
\text { Peak }: p_{i}(k) \leq P_{m}^{\text {mask }}(k) / N, \forall i, \forall k, \forall m .
$$

In summary, the problem is

$$
\begin{aligned}
& \max : V=\sum_{i=1}^{N} \sum_{k=1}^{K} \omega_{i} \log _{2}\left(1+\Gamma \frac{p_{i}(k)\left|g_{i, i}(k)\right|^{2}}{\xi_{i}(k)}\right) \\
& \text { s.t: } \\
& C_{i, \min } \leq \sum_{k=1}^{K} \log _{2}\left(1+\Gamma \frac{p_{i}(k)\left|g_{i, i}(k)\right|^{2}}{\xi_{i}(k)}\right), \forall i, \\
& \sum_{k=1}^{K} p_{i}(k) \leq p_{i, \max }, \forall i, \\
& p_{i}(k) \leq P_{m}^{\text {mask }}(k) / N, \forall i, \forall k, \forall m .
\end{aligned}
$$

\section{ITERATIVE WATER-FILLING BASED BARGAINING APPROACH}

For this problem itself, it is not an easily-solved problem. Taking into account the characteristics of cognitive network itself, multiple nodes are difficult to form a cooperative relationship. At the same time, we also expect the design algorithm is distributed, and the communication overhead should be small. Motivated by the work of [21]. We will adopt a decomposition approach to solve this problem. At the same time, in view of the multiple user iterative water-filling algorithm [22] has a number of excellent properties such as
Remark 1: Summarizing Remarks on Iterative Water Filling:

- The algorithm functions in a self-organized manner, thereby making it possible for the network to assume an ad-hoc structure.

- It avoids the need for communication links (i.e., coordination) among the multiple users, thereby significantly simplifying the design of the network.

- By using convex optimization, the algorithm tends to converge relatively rapidly to a Nash equilibrium; however, once this stable point is reached, no user is permitted to change its transmit-power control policy unilaterally.

- Computational complexity of the algorithm is relatively low, being on the order of two numbers: the number of secondary users and the number of spectrum holes available for utilization.

At the same time, the algorithm to obtain solutions proven to meet the requirements of the Nash equilibrium solution under only a few mild requirements. Through a rigorous mathematical derivation, we will adopt a joint approach to solve the above-mentioned two kinds of problems that we have defined.

\section{A. Decomposed Ordinal Problem}

For the original definition of the problem in (11), we use Lagrangian Layering Technology. Here, we first consider the peak interference-power constraints, since it is easily to deal with. Therefore, we should introduce the Lagrangian multiplier $\lambda$ and $v$ for the first and the second constraint conditions in (11). And we can get the mathematical expression after the relaxation as (12).

Here, $\Theta_{i}(k)=\left(\omega_{i}+\lambda_{i}\right) \log _{2}\left(1+\Gamma \gamma_{i}(k)\right)-v_{i} p_{i}(k)$.

In accordance with the decomposition theory, we have the multiple sub-problems,

$$
\begin{aligned}
& \max :\left\{\Theta_{i}(k)=\left(\omega_{i}+\lambda_{i}\right) \log _{2}\left(1+\Gamma \gamma_{i}(k)\right)-v_{i} p_{i}(k)\right\} \\
& s t: p_{i}(k) \leq P_{m}^{\text {mask }}(k) / N, \forall i, \forall k, \forall m .
\end{aligned}
$$

and, the dual master problem,

$$
\begin{aligned}
& \min :\left\{\Theta=\sum_{i=1}^{N} \sum_{k=1}^{K}\left\{\Theta_{i}^{*}(k)\right\}-\lambda^{T} C_{\min }+v^{T} p_{\max }\right\} \\
& \text { s.t. : } \lambda \geq 0, v \geq 0
\end{aligned}
$$

where, $\Theta_{i}^{*}(k)$ is optimal individual payoff with the capacity achieved by $S U_{i}$ as $C_{i}^{*}(k)=\log _{2}\left(1+\Gamma \gamma_{i}^{*}(k)\right)$ on the channel $k$ and the power consumed $p_{i}^{*}(k)$ into consideration. $\Theta_{i}^{*}(k)$ is achieved when each user obtains the optimal power level 


$$
\begin{aligned}
& \Theta=\sum_{i=1}^{N} \sum_{k=1}^{K} \omega_{i} \log _{2}\left(1+\Gamma \gamma_{i}(k)\right)-\sum_{i=1}^{N} \lambda_{i}\left(C_{i, \min }-\sum_{k=1}^{K} \log \left(1+\Gamma \gamma_{i}(k)\right)\right)-\sum_{i=1}^{N} v_{i}\left(\sum_{k=1}^{K} p_{i}(k)-p_{i, \max }\right) \\
& =\sum_{i=1}^{N} \sum_{k=1}^{K}\left\{\left(\omega_{i}+\lambda_{i}\right) \log _{2}\left(1+\Gamma \gamma_{i}(k)\right)-v_{i} p_{i}(k)\right\}-\lambda^{T} C_{\min }+v^{T} p_{\max } \\
& =\sum_{i=1}^{N} \sum_{k=1}^{K}\left\{\Theta_{i}(k)\right\}-\lambda^{T} C_{\min }+v^{T} p_{\max } .
\end{aligned}
$$

$p_{i}^{*}(k)$ and the best channel selection policy as the subproblem in (13).

\section{B. Iterative Water-Filling Approach For Subproblems}

Observe the subproblems in (13), we can see that it is a non-cooperative strategic game $G=\{N, S, U\}$.

Definition 1: Non-cooperative resource management game (NRMG): $G=\{N, S, U\}$, where $N$ is the player set, here it refers to the secondary transmitter-receiver pair. $S$ is the Cartesian product space consisted by $S_{i}, i=1, \ldots, N$. And $S_{i}$ is

$$
S_{i}=\left\{p_{i} \mid 0 \leq p_{i}(k) \leq p_{m}^{\text {mask }}(k) / N, \forall k \in K, \forall m \in M\right\} .
$$

Meanwhile, the individual payoff function is

$$
\left\{\Theta_{i}(k)=\left(\omega_{i}+\lambda_{i}\right) \log _{2}\left(1+\Gamma \gamma_{i}(k)\right)-v_{i} p_{i}(k)\right\}
$$

Here, Further, we derive the first-order partial difference function of individual payoff function in (13) with respect to $p_{i}(k), i=1, \ldots, N$. Then we obtain,

$$
\frac{\partial \Theta_{i}(k)}{\partial p_{i}(k)}=\frac{\omega_{i}+\lambda_{i}}{\ln 2} \frac{\Gamma}{1+\Gamma \gamma_{i}(k)} \frac{g_{i, i}(k)}{\xi_{i}(k)}-v_{i} .
$$

Let (15) equal to zero, and we can get the optimal power for the $S U_{i}$ at the channel $k$ is

$$
p_{i}^{*}(k)=\frac{\omega_{i}+\lambda_{i}}{v_{i} \ln 2}-\frac{\xi_{i}(k)}{\Gamma g_{i, i}(k)} .
$$

To observe the cooperation and the competition relationship of the multiple SUs, substitute $\xi_{i}(k)$ with into (16). Then we obtain the water-filling function as shown in (17).

$$
\left[\mathcal{W} \mathcal{F}_{i}\left(\mathbf{p}_{-i}\right)\right]_{k} \triangleq \kappa_{i}-\frac{\vartheta_{i}(k)}{g_{i, i}(k)}
$$

where $\quad \kappa_{i}=\frac{\omega_{i}+\lambda_{i}}{v_{i} \ln 2}, \quad$ and $\vartheta_{i}(k)$ $1 / \Gamma \sum_{j=1, j \neq i}^{N}\left(\sum_{k_{1}=1}^{K} \rho\left(k-k_{1}\right) p_{j}\left(k_{1}\right)\left|g_{j, i}\left(k_{1}\right)\right|^{2}\right)$ $\sum_{m=1}^{M}\left(\sum_{k_{1}=1}^{K} \rho\left(k-k_{1}\right) p_{m}\left(k_{1}\right)\left|h_{m, i}\left(k_{1}\right)\right|^{2}\right)+\sigma_{i}^{2}(k)$.

Here, we give a lemma for the existence and the uniqueness of Nash equilibrium solution for the NRMG model.

Lemma 1: The optimal solution $\mathbf{p}_{i}^{*}=\left\{p_{i}^{*}(k)\right\}_{k=1}^{K}$ for the proposed NRMG model exists and is unique. Meanwhile,

$$
\mathbf{p}_{i}^{*}=\mathcal{W F}_{i}\left(p_{1}, \ldots, p_{i-1}, p_{i+1}, \ldots, p_{N}\right)=\mathcal{W} \mathcal{F}_{i}\left(\mathbf{p}_{-i}\right)
$$

where the water-filling function is denoted as (17).

Definition 2: A feasible strategy profile $p^{*}=\left\{p^{*}\right\}_{i=1}^{N}$ is the NES of the NRMG model if

$$
U_{i}\left(p_{i}^{*}, p_{-i}^{*}\right) \geq U_{i}\left(p_{i}, p_{-i}^{*}\right), \forall p_{i} \in S_{i}, \forall i \in N .
$$

According to the Lemma 1, all the NES of the NRMG model need to satisfy the condition expressed by the following corollary.

Corollary 3: A feasible strategy profile $p^{*}=\left\{p^{*}\right\}_{i=1}^{N}$ is the NES of the NRMG model if and only if it salifies the following systems of nonlinear equation:

$$
\mathbf{p}_{i}^{*}=\mathcal{W} \mathcal{F}_{i}\left(\mathbf{p}_{1}^{*}, \ldots, \mathbf{p}_{i-1}^{*}, \mathbf{p}_{i+1}^{*}, \ldots, \mathbf{p}_{N}^{*}\right)
$$

with $\mathcal{W F}_{i}$ defined in (17).

More specifically, we propose two alternative totally distributed algorithms based on the water-filling solution in (7), and provide a unified set of convergence conditions for both algorithms. A. Sequential Iterative Water-Filling Algorithm (IWFA) The sequential IWFA we propose is an instance of the GaussíCSeidel scheme (by which, each userąís power level is sequentially updated [22]) based on the mapping (7): Each player, sequentially and according to a fixed updating order, solves problem (6), performing the single-user water-filling solution in (7). The sequential IWFA is described in Algorithm 1.

$$
\begin{aligned}
& \hline \hline \text { Algorithm 1: Sequential IWFA } \\
& \hline \text { 1. Set } \mathbf{p}^{(0)}=\left\{p^{(0)}\right\}_{i=1}^{N} \text {, here we carefully choose } \\
& \text { the initial power level } \mathbf{p}^{(0)} \text { with the feasible } \\
& \text { strategy set } S_{i} \text { in mind. } \\
& \text { 2. For } n=0: \text { numberofiterations, } \\
& p_{i}^{(n+1)}=\left\{\begin{array}{l}
\mathcal{W} \mathcal{F}_{i}\left(p_{-i}^{(n)}\right), \text { if }(n+1) \bmod N=i, \\
p_{i}^{(n)}, \text { otherwise }, \forall i \in N .
\end{array}\right. \\
& \text { 3. end. }
\end{aligned}
$$

To overcome the drawback of the possible slow speed of convergence, we consider in this section the simultaneous version of the IWFA, called simultaneous IWFA. The algorithm is an instance of the Jacobi scheme: At each iteration, the users update their own PSD simultaneously, performing the water-filling solution, given the interference generated by the other users in the previous iteration. The simultaneous IWFA is described in Algorithm 2.

\begin{tabular}{l}
\hline \hline Algorithm 2: Parallel IWFA \\
\hline 1. Set $\mathbf{p}^{(0)}=\left\{p^{(0)}\right\}_{i=1}^{N}$, here we carefully choose \\
the initial power level $\mathbf{p}^{(0)}$ with the feasible \\
strategy set $S_{i}$ in mind. \\
2. For $n=0:$ numberofiterations, \\
$p_{i}^{(n+1)}=\mathcal{W F}_{i}\left(p_{-i}^{(n)}\right), \forall i \in N$. \\
3. end.
\end{tabular}

\section{Gradient Projection Method For Master Problem}

For dual master problem denoted as (14), $\Theta_{i}^{*}(k)$ is best individual payoff function as above-mentioned, it is achieved 
by the proposed IWFA in the above subsections, when multiple users obtain the optimal transmission strategies. That is,

$$
\Theta_{i}^{*}(k)=\mathcal{F}\left(p_{i}^{*}(k), p_{-i}^{*}(k), \lambda_{i}, v_{i}\right)
$$

The optimal strategies $p_{i}^{*}(k), p_{-i}^{*}(k)$ in this step are constants. $\left[\Theta_{i}^{*}(k)\right.$ is the function of $\left(\lambda_{i}, v_{i}\right)$.

We employ the Gradient Projection Method for master problem,

$$
\lambda_{i}(t+1)=\lambda_{i}(t)+\alpha(t)\left(\bar{\lambda}_{i}(t)-\lambda_{i}(t)\right)
$$

where $\lambda_{i}$ is referred to the dual variables, $\alpha(t)$ is the iteration step. Such an iteration looks like a gradient projection method except that a subgradient is used instead of the gradient (which may not exist).

There are many results on convergence of the subgradient method. For constant step size $\alpha(t)=\alpha$ and constant step length $\alpha(t)=\alpha /\|G(t)\|$, the subgradient algorithm is guaranteed to converge to within some range of the optimal value; in other words, the subgradient method finds an $\epsilon$-suboptimal point within a finite number of steps. For the diminishing step size rule

$$
\alpha(t)=\frac{m+1}{m+t}
$$

where $m$ is a fixed nonnegative number, the algorithm is guaranteed to converge to the optimal value.

The gradient $\bar{\lambda}_{i}(t)$ of the master dual problem (21) is

$$
\bar{\lambda}_{i}(t)=\left[\lambda_{i}(t)+\left.s(t) \frac{\partial \Theta}{\partial \lambda_{i}}\right|_{\lambda_{i}=\lambda_{i}(t)}\right]
$$

where $s(t)$ is a scale at the $t s t$ iteration. Further,

$$
\frac{\partial \Theta}{\partial \lambda_{i}}=\sum_{k=1}^{K} \log _{2}\left(1+\Gamma \gamma_{i}(k)\right)-C_{i, \min } .
$$

For another dual variable, we have the similar representation as (25).

$$
\begin{aligned}
& v_{i}(t+1)=v_{i}(t)+\alpha(k)\left(\bar{v}_{i}(t)-v_{i}(t)\right) \\
& \bar{v}_{i}(t)=\left[v_{i}(t)+\left.s(t) \frac{\partial \Theta}{\partial v_{i}}\right|_{v_{i}=v_{i}(t)}\right]
\end{aligned}
$$

where $\frac{\partial \Theta}{\partial v_{i}}=p_{i, \max }-\sum_{k=1}^{K} p_{i}(k)$.

Here we have some further observations, which are given out using the remark form.

Remark 2: Further observations to the sub-problems and the dual master problem:

- We always can find the optimal policy $p_{i}(k), i \in N, k \in$ $K$, after the IWFA (17) obtain the optimal dual information, e.g., $\lambda_{i}(21)$.

- We note here that there are two algorithms, respectively, to solve the master problem using (21) and (25) and subproblems issues (17) after the successful decomposition of original problem (11).

- There exists the presence of information interaction, which is actually the strategy information $\lambda_{i}, v_{i}, p_{i}(k)$ interaction and does not include environmental information such as the complex and large amounts of information interaction process.
- These two algorithms will be gradually interactive policy data, the dynamic implementation will keep on in the finite steps and eventually achieve a balanced performance.

\section{IWFA-based Bargaining Approach}

Here, we propose a Nash bargaining structure shown as Fig.2,

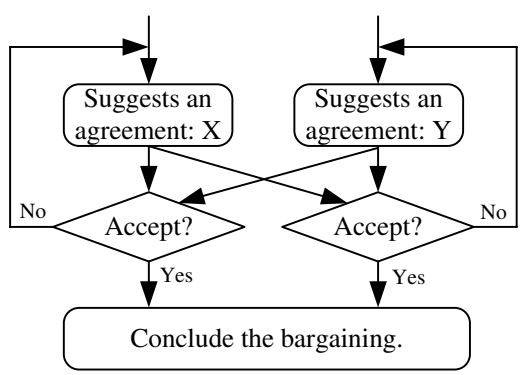

Fig. 2. Bargaining process, where there are only two players.

Fig.2 illustrates the bargaining concept. In the previous literature, design of algorithms is not well reflect the concept of game or a specific bargaining process. In this paper, we assume that the two players are $S U_{1}$ and $S U_{2}$.

As Fig. 2 shown, first, $S U_{1}$ proposes a transmission strategy $X$, then further asked another user $S U_{2}$ whether he is to accept such a strategy $X$. If $S U_{2}$ can accept the recommendations of the transmission strategy of $S U_{1}$, the bargaining process is over. Otherwise, when $S U_{1}$ understands the final strategy agreement hasn't been concluded, during the next bargaining process, the $S U_{2}$ will put forward his recommended strategy $Y$. Similarly, repeat the above process until the final agreement is conclude.

We can see that this process requires a lot of information exchange, interaction, resulting in communication overhead is too large. If there is no central control mechanism or a specialized agency, it is very difficult to complete. For multiuser scenario, this is more of a process can not be imagined. In addition to these, we also have some further observations and discussions on this bargaining process.

Remark 3: Many problems in the bargaining process need to be done.

- When the suggested policy of $S U_{1}$ isn't accepted by $S U_{2}$ in this circumstances, the adjustment strategy, or other player's strategy is what in the next bargaining step of the the proposed framework.

- If you have not been able to form a stable strategy after limit iterations, at this time, the user should use what kind of Transmission Strategy.

- What is decision-making mechanism to determine whether to accept the opponent's strategy.

To solve these problems, we have proposed IWFA-based bargaining approach. The detailed adjustment strategy is the Fig.2 in illustrated as Fig.3. 


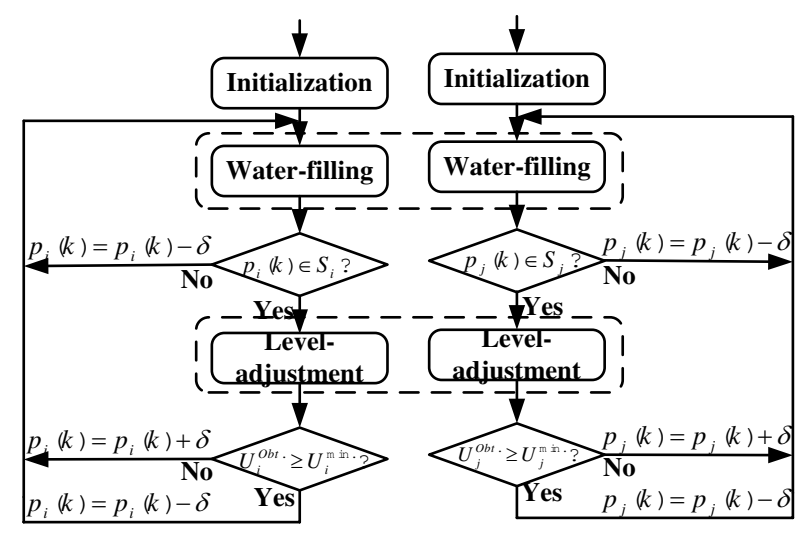

Fig. 3. Iterative water-filling based bargaining approach

\section{CONCLUSION}

Motivated by the cooperative game theory and the celebrated decomposition technique, the spectral gap-filling problem is formulated as cognitive capacity Nash product maximization $(C C N P M)$. The cooperation and competition behavior is reflected by the newly-described signal-to-interference plus noise (SINR) definition. From the Nash bargaining game, the spectrum gap filling problem is well discussed, which take multiple constraint conditions into consideration. It provides an uniform mathematical model for further studying the cooperative game theory for distributive approaches design. Using the decomposition technique, we proposed the distributed bargaining algorithm based on the celebrated iterative water-filling algorithm (IWFA), which maintain the typical distributed and simple-implementation properties. Specific conclusions are given by the mathematical proof and detail discussion.

\section{ACKNOWLEDGMENT}

This research was supported by National Science Fund for Distinguished Young Scholars (No.60725105), National Basic Research Program of China (No.2009CB320404), Program for Changjiang Scholars and Innovative Research Team in University, the 863 projects (No.2007A A01Z288), the National Nature Science Foundation of China (No.60572146), the Research Fund for the Doctoral Program of Higher Education (No.20050701007), the Teaching and Research Award Program for Outstanding Young Teachers in Higher Education Institutions of MOE, P. R. China, the Key Project of Chinese Ministry of Education (No.107103), the 111 Project (No.B08038), National S and T Major Project (2009ZX03007005), ISN090305.

\section{REFERENCES}

[1] Mitola J., Cognitive radio: An integrated agent architecture for software defined radio. Doctor of Technology, Royal Institute of Technology. Stockholm. Sweden. 2000.

[2] Haykin, Simon, Cognitive radio: Brain-empowered wireless communications, IEEE Journal on Selected Areas in Communications, Volume 23, Issue 2, February 2005 Page(s): 201-220.
[3] Zhou, Pan; Yuan, Wei; Liu, Wei, Joint power and rate control in cognitive radio networks: A game-theoretical approach, IEEE ICC 2008, pp: 3296-3301, 2008.

[4] Daoud, Ashraf Al; Alpcan, Tansu; Agarwal, Sachini, A Stackelberg game for pricing uplink power in wide-band cognitive radio networks, Proceedings of the 47th IEEE CDC 2008, pp: 1422-1427, 2008.

[5] Su, Yi; Van Der Schaar, Mihaela, A new perspective on multi-user power control games in interference channels, IEEE Transactions on Wireless Communications, v8, n6, pp: 2910-2919, June 2009.

[6] Su, Yi; Van Der Schaar, Mihaela, S-modular games for distributed power allocation in cognitive radio systems, IEEE Transactions on Wireless Communications, v8, n6, pp: 2910-2919, June 2009.

[7] Chungang Yang, Jiandong Li, A Game-Theoretic Approach to Adaptive Utility-Based Power Control in Cognitive Radio Networks, 2009 IEEE 70th Vehicular Technology Conference Fall VTC2009-Fall,20-23 Sept.,Anchorage, Alaska, USA.

[8] Scutari, G.; Barbarossa, S.; Palomar, D.P., Potential games: A framework for vector power control problems with coupled constraints, IEEE International Conference on Acoustics, Speech and Signal Processing - Proceedings, v4, pp: IV241-IV244, 2006.

[9] Wang, Fan; Krunz, Marwan; Cui, Shuguang, Price-based spectrum management in cognitive radio networks, IEEE Journal on Selected Topics in Signal Processing, v2, n1, pp: 74-87, February 2008.

[10] Rasti, Mehdi; Sharafat, Ahmad R.; Seyfe, Babak, Pareto-efficiency and goal-driven power control in wireless networks: a game-theoretic approach with a novel pricing scheme, IEEE/ACM Transactions on Networking, v17, n2, pp: 556-569, 2009.

[11] Chungang Yang, Jiandong Li, Distributed Power Control Method Based on the Nash Bargaining Solution from the Cooperative Game Theory for Cognitive Radio Networks, Journal of Beijing University of posts and telecommunications, in Chinese.

[12] Chungang Yang, Jiandong Li, and Zhi Tian, Optimal Power Control for Cognitive Radio Networks under Coupled Interference Constraints: A Cooperative Game-Theoretic Perspective, IEEE Transactions on Vehicular Technology, Special Issue on Cognitive Radio, 2010.

[13] Attar, Alireza; Nakhai, Mohammad Reza; Aghvami, A. Hamid, Cognitive radio game: a framework for efficiency, fairness and QoS guarantee, IEEE International Conference on Communications, p 4170-4174, 2008.

[14] Han, Zhu; Ji, Zhu; Liu, K.J. Ray, Fair multiuser channel allocation for OFDMA networks using Nash bargaining solutions and coalitions, IEEE Transactions on Communications, v53, n8, pp: 1366-1376, August 2005.

[15] Attar, Alireza; Nakhai, Mohammad Reza; Aghvami, A. Hamid, Cognitive Radio Game for Secondary Spectrum Access Problem, IEEE Transactions on Wireless Communications, v 8, n 4, p 2121-2131, April 2009.

[16] Maskery, Micheal; Krishnamurthy, Vikram; Zhao, Qing, Decentralized dynamic spectrum access for cognitive radios: Cooperative design of a non-cooperative game, IEEE Transactions on Communications, v57, n2, pp: 459-569, 2009.

[17] Maskery, Micheal; Krishnamurthy, Vikram; Zhao, Qing, Power control and channel allocation in cognitive radio networks with primary users cooperation, IEEE Transactions on Communications, v57, n2, pp: 459$569,2009$.

[18] Cendrillon, Raphael; Huang, Jianwei; Chiang, Mung, Autonomous spectrum balancing for digital subscriber lines, IEEE Transactions on Signal Processing, v55, n8, pp: 4241-4257, August 2007.

[19] Yaiche, Haikel; Mazumdar, Ravi R.; Rosenberg, Catherine, A game theoretic framework for bandwidth allocation and pricing in broadband networks, IEEE/ACM Transactions on Networking, v8, n5, pp: 667-678, Oct 2000.

[20] Schubert, Martin; Boche, Holgere, Nash bargaining and proportional fairness for log-convex utility sets, IEEE International Conference on Acoustics, Speech and Signal Processing - Proceedings, pp: 3157-3160, 2008.

[21] Palomar, Daniel P.; Chiang, Mung , A Tutorial on Decomposition Methods for Network Utility Maximization, IEEE Journal on Selected Areas in Communications, v24, n8, pp: 1439-1451, August 2006.

[22] Yu, Wei; Ginis, George; Cioffi, John M., Distributed multiuser power control for digital subscriber lines, IEEE Journal on Selected Areas in Communications, v20, n5, pp: 1105-1115, June 2002. 